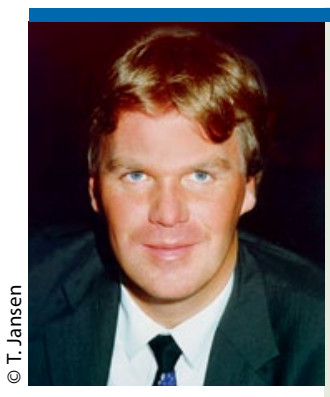

Priv.-Doz. Dr. Thomas Jansen Dermatologe in Köln

\title{
Akneentstehung und therapeutische Optionen
}

? Was weiß man über die Akneentstehung bei erwachsenen Frauen? Kommen die Pickel vom Stress, von den Hormonen oder von der schlechten Ernährung?

Jansen: Grundsätzlich gilt das Gleiche wie für die Akne bei Jugendlichen, nur müssen die einzelnen pathogenetischen Faktoren anders gewichtet werden. Eine genetische Prädisposition scheint zu bestehen. Diese wird beeinflusst durch innere Faktoren, zum Beispiel Hormonstörungen oder Stress, und äußere Faktoren wie Kosmetika, Medikamente, Ernährung oder Rauchen. An ein polyzystisches Ovarialsyndrom - PCOS muss man denken, wenn neben der Akne andere Zeichen der Hyperandrogenämie vorhanden sind: Hirsutismus, androgenetische Alopezie, Zyklusstörungen und auch Übergewicht. Stress hat einen modulierenden Einfluss auf Akne, das ist heute nicht mehr zu leugnen. Außerdem muss man Medikamente abfragen, die eine vorbestehende Akne verschlechtern können. Dazu zählen als Kontrazeptiva eingesetzte Gestagene mit androgener Wirkung.

? Stichwort Ernährungsberatung: Worauf sollten Betroffene konkret verzichten?

Jansen: Die Ernährungsberatung hat angesichts der Datenlage einen besonderen Stellenwert. Es gibt zahlreiche Hinweise auf einen Zusammenhang von Akne und westlicher Ernährung. Diese ist durch hyperglykämisch wirkende Kohlenhydrate, durch Milch und Milchprodukte und auch durch entzündungsfördernde Fettsäuren gekennzeichnet. Auch Süßigkeiten haben einen negativen Einfluss auf die Akne. Konkret heißt das: weniger Fast Food und Süßigkeiten, vor allem weniger Milchschokolade, zuckerhaltige Limonaden und tierische Fette mit hohem Gehalt an Arachidonsäure. Wichtig ist auch die Abstinenz vom Rauchen. Dieses kann durch Überflutung der Gesichtshaut mit chlorierten Kohlenwasserstoffen zu einer nicht entzündlichen zystischen Akne führen. Es fehlen jedoch bisher prospektive kontrollierte Studien, die den Nutzen dieser Maßnahmen belegen.
? Worin unterscheidet sich die Therapie der Erwachsenenakne von der bei jugendlicher Pickelhaut?

Jansen: Grundsätzlich gelten die Therapiestandards, die für die Pubertätsakne entwickelt wurden, auch bei erwachsenen Frauen. Das Behandlungsprinzip weicht aber in einigen Punkten davon ab. Der Langzeit- oder Erhaltungstherapie mit Prävention von Mikrokomedonen kommt eine besondere Bedeutung zu. Als topische Wirkstoffe sind hierbei Retinoide, insbesondere Adapalen, und Azelainsäure geeignet. Azelainsäure ist auch zur Aknebehandlung in Schwangerschaft und Stillzeit einsetzbar, wenn eine Indikation gegeben ist. Bei therapieresistenter oder rezidivierender Akne kommen eine niedrig dosierte systemische Langzeitbehandlung und moderat dosierte intermittierende Behandlungen mit Isotretinoin in Betracht. Bei den Hormonbehandlungen sind Antiandrogene, die in kombinierten Kontrazeptiva enthalten sind, am wirksamsten. Bei Nachweis eines PCOS ist der Insulinsensitizer Metformin die Therapie der Wahl, sein Einsatz erfolgt jedoch "off-label".

? Was muss man in puncto Nebenwirkungen beachten?

Jansen: Aus Studien weiß man, dass nur ein geringer Teil der erwachsenen Frauen mit Akne deswegen zum Arzt geht, und zwar aus Angst vor schlechter Verträglichkeit der topischen Aknetherapeutika. Häufig wird als Grund auch die mangelnde Wirksamkeit systemischer Medikamente angegeben. Die reife Haut erwachsener Frauen ist vielfach empfindlicher als die Haut von Jugendlichen, daher ist auf gute Verträglichkeit zu achten. Bei Isotretinoin muss man beachten, dass die Substanz unabhängig von der Dosis teratogen wirkt. Bei Frauen im gebärfähigen Alter sind also strikte Sicherheitsmaßnahmen einzuhalten, das heißt Schwangerschaftsausschluss und sichere Kontrazeption!

! Herr Dr. Jansen, wir danken für das Gespräch. bung eines Kontrazeptivums theoretisch zwei Fliegen mit einer Klappe. Die „Pille“ sollte neben Ethinylestradiol eines der neueren Gestagene enthalten, z.B. Desogestrel, Gestoden, Levonorgestrel oder Norgestimat. Besser als diese scheinen Präparate mit Cyproteronacetat zu wirken. Diese Substanz hat als Bestandteil des Akne- und (Off-label)-Verhütungsmittels Diane-35 kürzlich für Schlagzeilen gesorgt (s. Seite 132).

\section{Die "schweren Geschütze"}

der Aknetherapie

Bei akut aufflammender Akne oder zur Kurzzeittherapie schwerer Formen könne man auf Glukokortikoide in niedrigen Dosierungen zurückgreifen, schreiben Dréno und Kollegen. Für solche schweren Fälle komme auch eine intermittierende orale Therapie mit Isotretinoin infrage, dieses werde aber meist erst dann eingesetzt, wenn andere Strategien 